\title{
FINGER TOUCH BASED ECG MONITORING
}

\author{
S.A.Benade ${ }^{1}$, U.L.Bombale ${ }^{2}$ \\ ${ }^{1}$ M.Tech.Student, Electronics Department, Shivaji University, Kolhapur, Maharashtra, India \\ ${ }^{2}$ Assistant Professor, Electronics Department, Shivaji University, Kolhapur, Maharashtra, India
}

\begin{abstract}
Since few years, study discloses that the evaluation of Electrocardiography (ECG) signals offers important advantage for biometric field. Many heart attacks can be judged and prevented by ECG waveform analysis. In health care centers, there is increase in the number of biomedical equipment used at hospital and home, but those lack in ability to provide handheld ECG monitor. In this paper, finger based ECG monitor system has been adopted. Instead of six electrodes, only two electrodes attached to fingers can give useful ECG waveform.ECG waveform can be displayed on thin film transistor i.e. TFT and personal computer i.e. PC. Advanced RISC machine, Instrumentation amplifier, ECG electrode and ECG signal conditioning, thin film transistor (TFT),ARDUINO board are included in hardware design. ARM ADC programming, TFT and ARDUINO programming and MATLAB programming for PC interfacing have been implemented in software design. Patient can touch the electrodes through fingers and his/her ECG will be displayed on TFT. Same ECG signal can be transferred to PC via USB cable. Investigational outcomes revealed that such a handheld ECG machine is appropriate in results, easy to use, and light weight.
\end{abstract}

Keywords: ARM, Instrumentation Amplifier, TFT, Electrode, ARDUINO, MATLAB

\section{INTRODUCTION}

The word electrocardiography is derived from Greek word Kardia which means Heart. ECG that is electrocardiography is a process of analysis of heart activity over the period of time and is sensed by electrodes attached to the body skin. An ECG is used to measure the heart's electrical conduction system. It picks up electrical impulses produced by the polarization and depolarization of cardiac tissue and converts into a waveform. The waveform is then used to measure the rate and consistency of heartbeats, as well as the size and position of the chambers, the presence of any damage to the heart, and the effects of the drugs or devices used to normalize the heart.

A typical ECG tracing of a normal heartbeat (or cardiac cycle) consists of a $\mathrm{P}$ wave, a QRS complex and a $\mathrm{T}$ wave (Figure 1). The baseline voltage of the electrocardiogram is known as the isoelectric line. Normally the isoelectric line is measured as the portion of the tracing following the $\mathrm{T}$ wave and prior the next $\mathrm{P}$ wave.

In medicine, especially cardiology, the QT interval is a measure of the time between the start of the $\mathrm{Q}$ wave and the end of the $\mathrm{T}$ wave in the heart's electrical cycle. The QT interval commonly represents electrical depolarization and repolarization of the left and right ventricles.

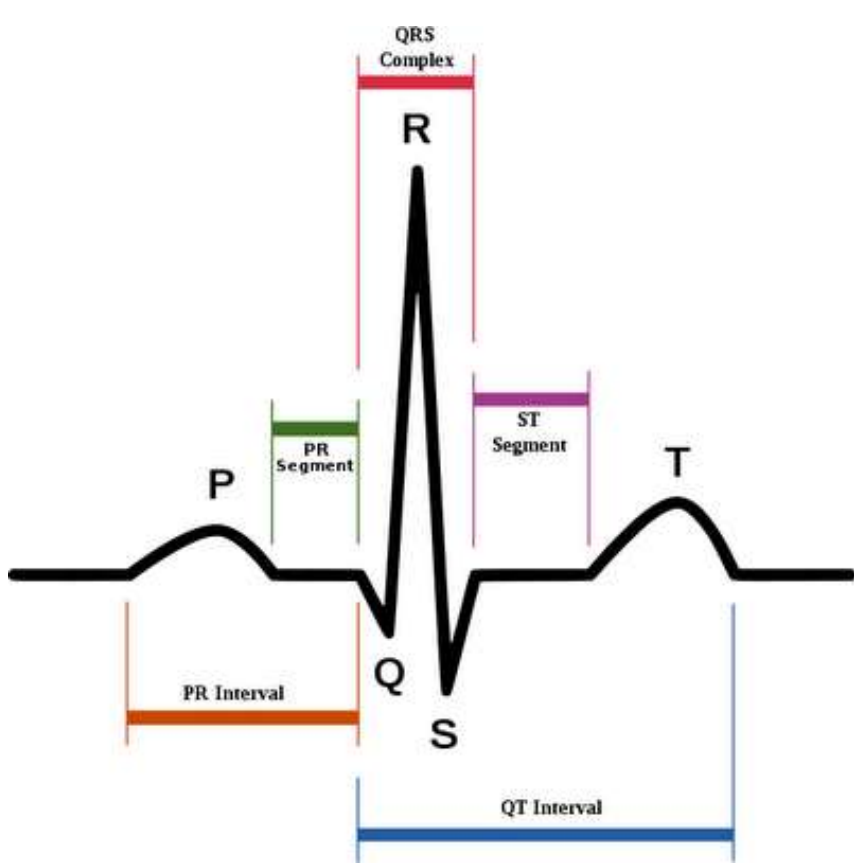

Fig-1: ECG waveform

To the clinician, the shape and duration of each feature of the ECG are significant. The waveform, however, depends greatly upon the lead configuration used. In general, theCardiologist looks critically at the various time intervals, polarities, and amplitudes to arrive at his diagnosis. Some normal values for amplitudes and durations of important ECG parameters are as follows:-

AMPLITIUDE:-

P wave:- $0.25 \mathrm{mv}$ - (Atrial Depolarization)

R wave: - $1.60 \mathrm{mv}$

Q wave: - $25 \%$ of $R$ wave 
T wave: - 0.1 to $.5 \mathrm{mv}-($ Ventricular Repolarization)

DURATION:-

P-R interval: - 0.12 to $0.20 \mathrm{sec}$

Q-T interval: - $0.35-0.44 \mathrm{sec}$

S-T segment: - 0.05-0.15 sec

$P$ wave interval: - $0.11 \mathrm{sec}$

QRS interval: - $0.09 \mathrm{sec}$ (Ventricular Depolarization)

\section{LITERATURE REVIEW}

Study discloses that among all the diseases, the three main causes accounting for the rise in death rate are cardiovascular disease [5],[6] Cancer and Kidney failure. Even though heart attack can happen abruptly without obvious indications, cardiac beatdisorders can often be found before the event. They can likely be utilized as the pioneer to main cardiac incidents [1].

Electrocardiography is a medicinal diagnostic technique used to store the electrical activity of the heart and show it as a waveform. An electrocardiogram (ECG) is obtained by measuring electrical potential between various points of thehuman body using an instrumentation amplifier attached to the body through leads attached to electrodes (electrical contacts). Electrodes are placed on different sides of the heart to measure the movement of many parts of the heart muscle and the voltage between pairs of these electrodes is what is returned as ECG in the form of a graph. Over traditional ECG machines, handheld pocket ECG monitor will be helpful. With rapid development in technology, some other ECG products are available using technologies like internet solutions [2], [8,] Bluetooth [3] wireless network [4],[7],[11].But it has problems of high cost and high power consumption.Wearable ECG system with PCB on $\mathrm{T}$ shirt is designed prior but it has to be worn all the time for ECG monitoring which is not comfortable. [10]

To solve above mentioned problems, we have developed an ECG monitor device in which patient will just touch electrodes through fingers and his/her ECG will be displayed alive on TFT screen. Same ECG will be observed on PC by connecting USB cable.

\section{IMPLEMENTATION}

\subsection{Hardware Implementation}

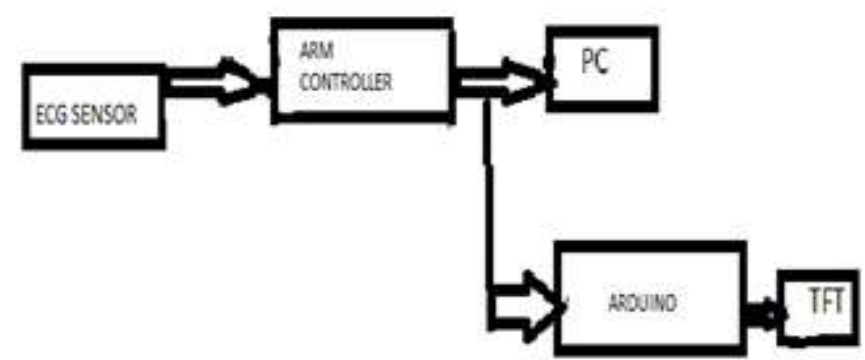

Fig -2: Block Diagram of Proposed system

Hardware consists of three main sections. First unit consists of ARM LPC 2138 along with sensor. Complete board of ECG sensor has three sensing probes of silver chloride, instrumentation amplifier, and filters. Output of sensor is analog voltage .This output is connected to ADC channel of ARM controller to read the data. The data is collected in array and then transmitted serially out using UART 1 port of ARM to PC. Same data is transmitted to Arduino board to display it on graphical LCD i.e.TFT.For any bio signal application, bio signal acquisition and preprocessing is required.[9].

\subsubsection{ECG sensor and ECG Processing Circuit}

Electrodes are ECG sensors i.e.it converts physical parameter to electrical signal. Electrodes pick up the ECG signal from body. It acts as a transducer.Biomedical signals are converted in to electrical signals. Normally twelve electrodes are used to detect ECG signals and those are attached to various parts of body. Gel is applied on body skin surface for getting better conductivity and then electrodes are placed using leads. Actually gel is quite irritating for skin and can create allergic reactions. But in this project instead of traditional twelve electrodes only three electrodes are used and gel is not required. Three sensing probes of Silver Chloride work as ECG sensors. The silver/silver chloride reference electrode is composed of a silver wire $(\mathrm{Ag})$ that has been coated with a layer of solid silver chloride $(\mathrm{AgCl})$, immersed in a solution that is saturated with $\mathrm{KCl}$ and $\mathrm{AgCl}$.

ECG signals vary from microvolt $(\mu \mathrm{V})$ to millivolt $(\mathrm{mv})$ range. It has to be converted in to suitable voltage level before applying it to digital circuit. Therefore ECG processing circuit is needed.For better implementation Instrumentation amplifier can be used.

\subsubsection{Design of Instrumentation Amplifier}

Operational amplifier INA 122 is used as an instrumentation amplifier. Gain of instrumentation amplifier INA 122 can be set by external resistor ' $\mathrm{Rg}$ ' connected between it's pin no. 1 and 8.It's gain formula:

$$
\mathrm{G}=5+200 \mathrm{~K} \Omega / \mathrm{Rg}
$$

ECG signal has bandwidth of $0.05 \mathrm{~Hz}$ to $150 \mathrm{~Hz}$.and it suffers from various noises. For filtering noises, various filters should be taken in to account in analog circuit.

\subsubsection{Design of High Pass Filter}

Baseline noise affects ECG signal and it has frequency of $0.5 \mathrm{~Hz}$ so High pass filter is designed to pass all frequency components above $0.5 \mathrm{~Hz}$.

\subsubsection{Design of Notch Filter}

Power line noise has frequency of $50 \mathrm{~Hz}$.In order to remove it, Notch filter is designed.

\subsubsection{Design of Low Pass Filter}

ECG signal has bandwidth $0.05 \mathrm{~Hz}$ to $150 \mathrm{~Hz}$ so Low pass filter with cut off frequency $150 \mathrm{~Hz}$ is designed to pass all frequency components below $150 \mathrm{~Hz}$. 


\subsection{Digital Circuit}

ARM7TDMI LPC 2138 is chosen. It has $512 \mathrm{~KB}$ on chip flash, 32Kb SRAM, two 8 channel 10 bit ADCs with conversion time $2.44 \mathrm{micro}$ seconds /channel.

ECG signal after processing is given to ADC0 channel ' 4 ' of ARM so analog ECG signal is converted into digital by ADCand it is serially transmitted to UART1.UART 1 is connected to ARDUINO UNO board and PC The ARDUINO UNO is an open source prototyping platform which is based on microcontroller ATmega328 It has 16 $\mathrm{MHz}$ clock speed, 32Kb flash and 2Kb SRAM, 14digital pins out of which 6 arePWM pins.

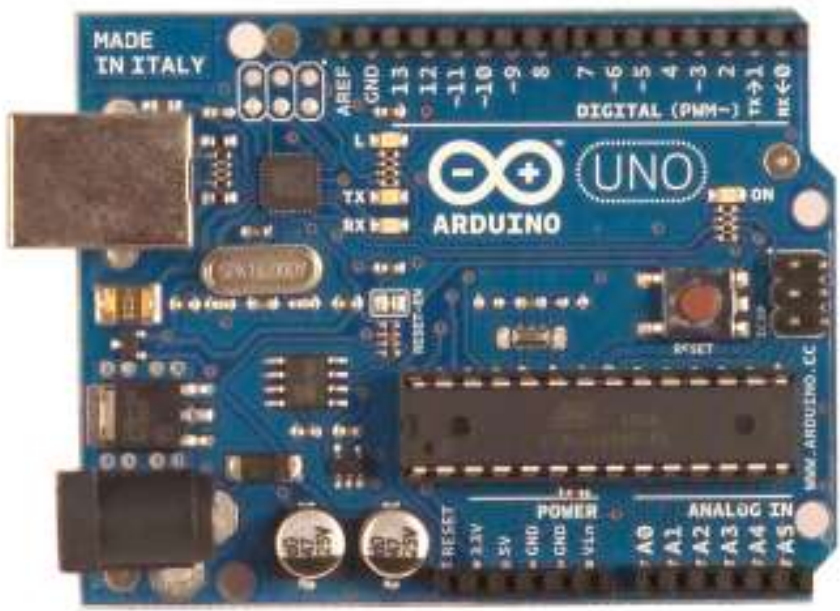

Fig-3: ARDUINO UNO board

On ARDUINO UNO board, 2.4"'diagonal thin film transistor (TFT) display is mounted. TFT resolution is $240 * 320$. TFT is Colorful, it has 18 bit 262,000 different shades and 4 wire resistive touch screen

ARDUINO board's all analog and digital pins, power, ground pins are connected to corresponding pins of TFT. So when ECG signal comes through electrodes it will be simultaneously displayed on TFT and PC. For TFT programming using Arduino, Adafruit graphics library and TFT specific library have to be included.

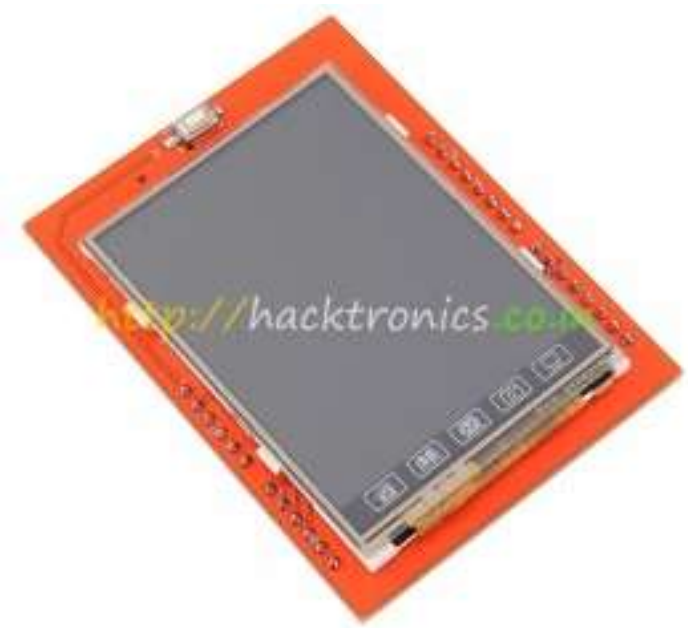

Fig-4: Thin film transistor (TFT)
ECG signal which is applied to ARDUINO board and TFT is also applied to PC using serial port. Therefore same ECG signal is displayed on PC simultaneously. For PC interfacing of ECG signal,MATLAB programming is done. In MATLAB, ECG array is defined to get values and then it is plotted till system gets interrupted.

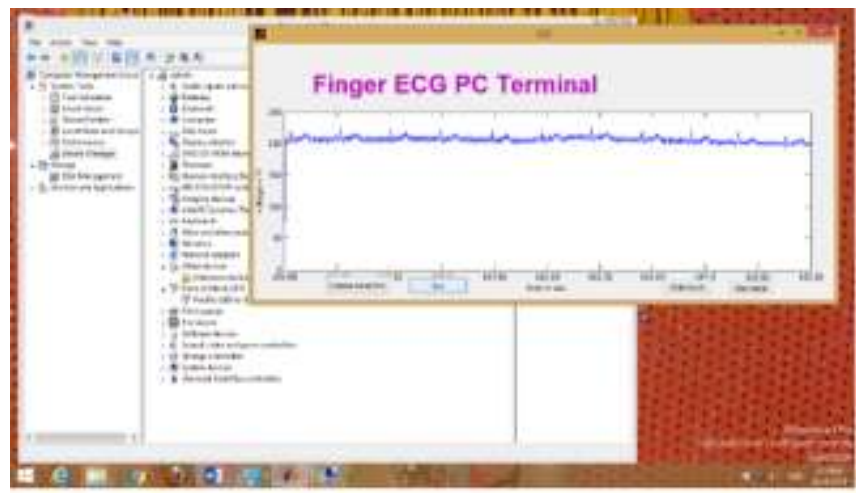

Fig-5: ECG captured on Personal Computer

For testing purpose,ECG signal was obeserved on Digital Storage oscilloscope(DSO) before applying to TFTand PC.Fig.6 indicates ECG waveform obesrved on DSO

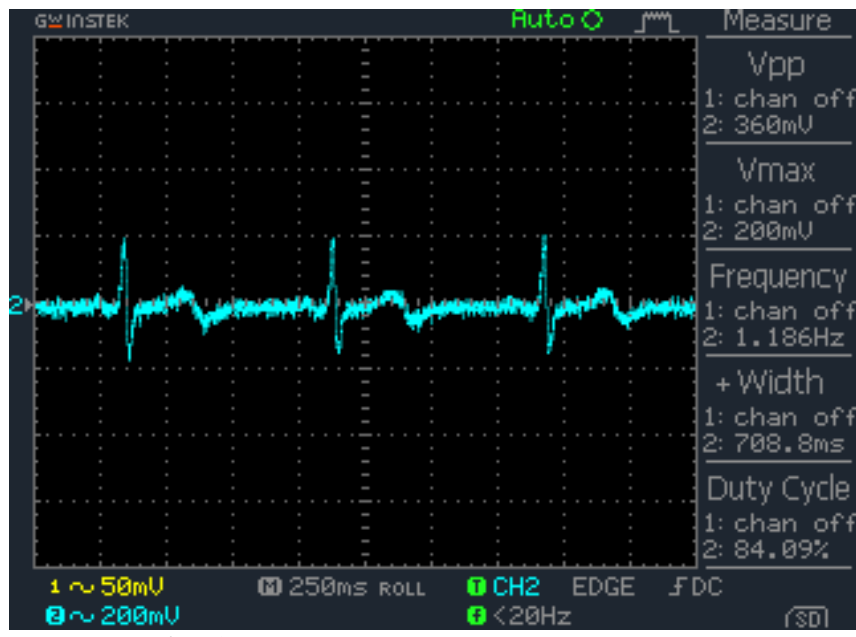

Fig-6:ECG obesrved on DSO for testing

Fig.7 indicates overall system design including ECG sensors, ARM board and TFT where ECG is displayed on TFT.

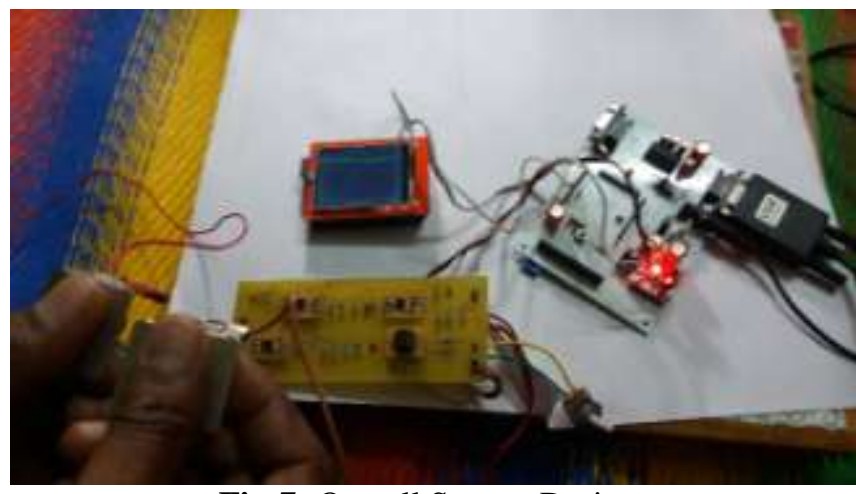

Fig-7: Overall System Design 


\section{FUTURE DEVELOPMENT}

For further development of project, normal ECG database of all age categories can be taken in memory andbuzzer will be interfaced to ARM. Displayed ECG waveform on TFT can be compared to database. If there is difference then buzzer will sound indicating that ECG waveform is not normal.

In this way, if any abnormal ECG is observed then it will be detected. Hence necessary diagnosis will be done and human life will be saved. The same circuit can be useful to detect Heart Beat rate using ECG waveform.

\section{CONCLUSION}

Sensing electrodes of silver chloride were used for picking ECG signals from right and left thumb fingers. As ECG signal is in micro volt to milli volt rang, amplification of ECG was carried out using instrumentation amplifier. ARM ADC requires 3.3 volt input, therefore considering this, proper gain was chosen before applying ECG to ARM.ECG has specific range and it gets contaminated by noise easily so noise filtering was carried out. LPC 2138 was chosen for processing ECG signal.ADC of same controller converts analog ECG signal in to Digital.For testing purpose ECG signal was observed on Digital storage oscilloscope before applying to TFT.For proper ECG display, TFT of $240 * 320$ resolution was used.As TFT libraries for ARM were not available,ARDUINO Uno board was chosen to serve as driver,hence ARM was interfaced to ARDUINO and ARDUINO was interfaced to TFT.For PC interfacing MATLAB was chosen .In MATLAB array was defined to get ECG .In this way, proper ECG waveform was captured on TFT and PC successfully.

\section{ACKNOWLEDGEMENT}

The author thankfully acknowledge to respected project guide Dr.U.L.Bombale (Assistant Professor Electronics department, Department of Technology, Shivaji University, Kolhapur), Coordinator Mr.P.C.Bhaskar (Assistant Professor, Electronics department, Department of Technology, Shivaji University, Kolhapur), Director Dr.J.S.Bagi Sir (Department of Technology, Shivaji University, Kolhapur), Dr.Mehata, (M.D.Medicine, Kolhapur)who helped me for completion of my project.

\section{REFERENCES}

[1]. India-Heart capital a paper by Mission Hospital Durgapur.PANIDIS IP and MORGANROTH J., Sudden death in hospitalized patients: cardiac rhythm disturbances detected by ambulatory electrocardiographic

[2]. A. I. Hernndez, F. Mora, G. Villegas, G. Passariello, and G. Carrault,"Real-Time ECG Transmission via Internet for Nonclinical Applications,"IEEE Transactions on Information Technology in Biomedicine,

[3]. J. Andreasson, M. Ekstrom, A. Fard, J. G. Castano, and T. Johnson, "Remote System for Patient Monitoring Using Bluetooth," Sensors, Proceedings
[4]. R. H. Istepanian, B. Woodward, E. Gorilas, and P. A. Balos, "Design of mobile telemedicine systems using GSM and IS-54 cellular telephone standards," J Telemed Telecare, Royal Society Of Medicine Press, England,vol. 4, pp. 80-82, 1998.

[5]. World Health statistics (WHO) 2013

[6]. Cardiovascular diseases in India Challenges and way ahead anInternational heart protection summit September 2011

[7]. D. L. Rollins, C. R. Killings worth, G. P. Walcott, R. K. Justice, and R.E. Ideker, "A Telemetry System for the Study of Spontaneous Cardiac Arrhythmias," IEEE transactions on bio-medical engineering, Institute ofElectrical and Electronics Engineers, USA, vol. 47, pp. 887-92, 2000

[8]. " Remote Electrocardiogram Monitoring Based On The Internet" Khalid Mohamed Alajel*, Khairi Bin Yosuf, Abdul Rhman Ramli,Department of Computer \& Communication System Engineering Faculty of Engineering, University Putra Malaysia 43300 UPM, Serdang, Selangor DE, MALAYSIA, KMITL Sci. J. Vol. 5 No.2Institute of Electrical and Electronics Engineers, USA, vol. 5, pp. 253-257, 2001

[09]. Rigas, et.al, “A User Independent, Biosignal Based, Emotion Recognition Method," Lecture notes in Computer Science, No.4511, pp.314-318, 2007

[10]. J. You, et.al, "A Wearable ECG Acquisition System With Compact Planar-Fashionable Circuit Board- Based Shirt," IEEE Transactions on Information Technology ni Biomedicine, Vol.13, pp.892-902, 2009.

[11]. A. C-W. Wong, et al, "A 1V Micropower System onChip for Vital-Sign Monitoring in Wireless Body Sensor Networks," ISSCC Dig. Tech. Papers, Pp.138-139, Feb., 2008

\section{BIOGRAPHIES}

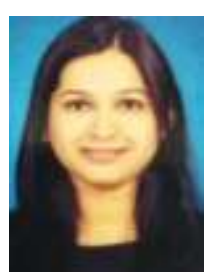

Miss. Sheetal Appasaheb Benade, M. Tech. Student, Electronics department, Shivaji University, Kolhapur, Maharashtra, India

Email Id: sheetal8686@gmail.com

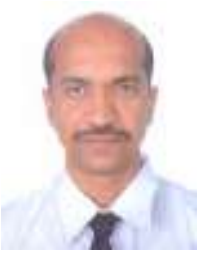

Dr.U.L.Bombale, Assistant Professor, Electronics department, Shivaji University, Kolhapur, Maharashtra, India Email Id: uttam_bombale@rediffmail.com 